\title{
Transurethral microwave treatment for benign prostatic hypertrophy: a randomised controlled clinical trial
}

\author{
A S Bdesha, C J Bunce, J P Kelleher, M E Snell, J Vukusic, R O’N Witherow
}

\begin{abstract}
Objectives-To determine whether transurethral microwave treatment for patients with benign prostatic hypertrophy provides significant symptomatic relief, a reduction in residual urine volumes, and improvements in flow rates compared with sham treatment.
\end{abstract}

Design-Prospective double blind randomised study with follow up at three months. teaching hospital.

Patients-40 men completed the study: 22 received microwave treatment and 18 received sham treatment. Entry criteria were symptoms of prostatism of at least six months' duration, a total symptom score $>14$, and a peak urine flow rate $<15 \mathrm{ml} / \mathrm{s}$ or a residual urine volume $>50 \mathrm{ml}$. Exclusion criteria were prostatic cancer, a residual urine volume $>200 \mathrm{ml}$, a very large prostate, an obstructing middle lobe, acute urinary retention, impaired renal function, coexisting urinary tract disease, and previous prostatic surgery.

Interventions-A single 90 minute transurethral microwave treatment or sham treatment.

Outcome measures-Patients' symptoms (including daytime frequency and nocturia) recorded in a self assessment symptom score questionnaire, peak urinary flow rates, and residual urine volumes.

Results-The mean total symptom scores of the patients who received microwave treatment fell from 30 to 11 compared with a fall from 31 to 26 for patients who received sham treatment $(p<0 \cdot 001)$. Among patients who received microwave treatment daytime frequency fell from 9.4 to 5.5 voids a day and night time frequency from 3.5 to 1.6 voids a night; residual urine volumes fell from $104 \mathrm{ml}$ to $52 \mathrm{ml}$; and peak urine flow rates increased by $2.3 \mathrm{ml} / \mathrm{s}$. In the control group there was no improvement in any of these features. Treatment preserved sexual function and antegrade ejaculation.

Conclusions-For selected patients with prosta-

Department of Urology, St Mary's Hospital, London W2 1NY A S Bdesha, registrar C J Bunce, research registrar J P Kelleher, senior registrar M E Snell, consultant urologist

RO'N Witherow, consultant urologist

\section{Laser Electro Optics, London SE25 \\ JVukusic}

Correspondence to: Mr Witherow.

BMF 1993;306:1293-6

\section{Introduction}

Prostatism is common with one in 10 men in their 40 s destined to undergo prostatectomy ${ }^{12}$ and seven in 10 men in their 60 s likely to suffer prostatic enlargement. ${ }^{3}$ Transurethral prostatectomy is usually a safe and effective treatment for benign prostatic hypertrophy, most studies reporting mortality of less than $1 \%^{4}$ and symptomatic improvement in $75 \%$ of patients. ${ }^{5-7}$ This, however, still leaves a quarter of patients dissatisfied with the outcome of their treatment, and side effects do occur though they are seldom severe. ${ }^{7-9}$ These problems have led to the development and investigation of medical and minimally invasive
Setting-Department of Urology in a London

treatments including antiandrogens, ${ }^{10} \alpha$ adrenergic blockade, ${ }^{11}$ balloon dilatation of the prostatic urethra, ${ }^{1213}$ intraurethral stents, ${ }^{14} \quad 5 \alpha$-reductase inhibitors, ${ }^{15}$ and microwave hyperthermia. ${ }^{16-22}$

Microwave hyperthermia is attractive because it can be performed on outpatients under local anaesthesia. In trials of multiple transrectal ${ }^{16}{ }^{17}$ and transurethral ${ }^{18-20}$ treatments patients showed good symptomatic improvement. Valid criticisms of these studies were that patients required multiple treatments and that all the series were uncontrolled so that the placebo effect was not assessed. A further criticism of microwave treatment is that it is being introduced into clinical practice without having been subjected to the same rigorous review that any new drug would undergo.

To answer these criticisms we performed a prospective randomised controlled trial with one group of patients receiving a single transurethral microwave treatment while the control group received a sham treatment.

\section{Patients and methods}

Before starting the study we obtained the local ethical committee's approval, and patients gave their full written informed consent before entering the study. We recruited patients who had had symptoms of prostatism for at least six months and who would normally have been offered a prostatectomy. We asked them if they would take part in a study to evaluate a new treatment. We told them that they might receive a sham treatment but that, if they did, they would subsequently have the option of microwave treatment or a conventional prostatectomy. Patients who agreed to take part completed a self assessment questionnaire based on the World Health Organisation's symptom score questionnaire. This recorded daytime frequency, and nocturia as absolute values; the force of the stream on a scale from 0 (no restriction) to 4 (severe restriction); and symptoms of hesitancy, terminal dribble, urgency, dysuria, intermittent voiding, and incomplete voiding on a scale from 0 (absence of the symptom) to 6 (symptom present all the time). Only patients whose scores were more than 14 were eligible to enter the study.

We performed a full clinical examination, including measured blood concentrations of prostate specific antigen and creatinine. Those patients with a raised antigen concentration or a suspicious nodule detected on rectal examination underwent transrectal ultrasonography and biopsy to check for carcinoma. We estimated the patients' residual urine volumes by measuring the dimensions of their bladders with ultrasonography before and after voiding. We calculated the patients' peak rates of urine flow by averaging at least two measurements of peak flow rates for voided volumes of at least $150 \mathrm{ml}$. Patients had to have a 
Mean (95\% confidence intervals) symptom scores, peak urine flow rates, and residual urine volumes of patients with benign prostatic hypertrophy given microwave treatment or sham treatment

\begin{tabular}{|c|c|c|c|c|c|}
\hline & \multicolumn{2}{|c|}{ Microwave treatment $(n=22)$} & \multicolumn{2}{|c|}{ Sham treatment $(n=18)$} & \multirow[b]{2}{*}{ p Value } \\
\hline & Before & After & Before & After & \\
\hline \multirow{12}{*}{$\begin{array}{l}\text { Total symptom score } \\
\text { Daytime frequency } \\
\text { Nocturia } \\
\text { Force of stream } \\
\text { Hesitancy } \\
\text { Terminal dribble } \\
\text { Urgency } \\
\text { Intermittent voiding } \\
\text { Incomplete voiding } \\
\text { Peak urine flow rate } \\
\text { (ml/s) } \\
\text { Residual urine volume } \\
\text { (ml) }\end{array}$} & $30(25.2$ to 34.8$)$ & $11(7.4$ to 14.6$)$ & $31(25.5$ to 36.5$)$ & $26(20.6$ to 31.4$)$ & $<0.001$ \\
\hline & $9.4(7.3$ to 11.5$)$ & $5.5(4.5$ to 6.5$)$ & $7 \cdot 4(5.4$ to 9.4$)$ & $7.4(5.9$ to 8.9$)$ & $<0.002$ \\
\hline & $3.5(2.6$ to 4.4$)$ & $1.6(0.9$ to 2.3$)$ & $3.5(2.5$ to 4.5$)$ & $3.3(2.9$ to 3.7$)$ & $<0.01$ \\
\hline & $2.6(2.0$ to 3.2$)$ & $1.0(0.6$ to 1.4$)$ & $2.2(1.7$ to 2.7$)$ & $2.2(1.8$ to 2.6$)$ & $<0.001$ \\
\hline & $2.5(1.6$ to 3.4$)$ & $0.9(0.6$ to 1.2$)$ & $2.6(1.8$ to 3.4$)$ & $1.7(1.0$ to 2.4$)$ & NS \\
\hline & $3.5(2.5$ to 4.5$)$ & $0.7(0.2$ to 1.2$)$ & $3.9(2.8$ to 5.0$)$ & $3.6(2.7$ to 4.5$)$ & $<0.02$ \\
\hline & $4.0(3.2$ to 4.8$)$ & $1.1(0.4$ to 1.8$)$ & $2.6(1.5$ to 3.7$)$ & $1.7(0.9$ to 2.5$)$ & $<0.05$ \\
\hline & $2.3(1.4$ to 3.2$)$ & $0.9(0.3$ to 1.5$)$ & $2.8(1.8$ to 3.8$)$ & $2.3(1.3$ to 3.3$)$ & NS \\
\hline & $2.7(1.6$ to 3.8$)$ & $0.7(0$ to 1.4$)$ & $2.5(1.5$ to 3.5$)$ & $2.0(1.0$ to 3.0$)$ & NS \\
\hline & & & & & \\
\hline & $12.3(10.7$ to 13.9$)$ & $14.6(12 \cdot 1$ to $17 \cdot 1)$ & $10 \cdot 8(9 \cdot 2$ to $12 \cdot 4)$ & $9.8(8.5$ to 11.1$)$ & NS \\
\hline & 104 (85 to 125$)$ & $52(34$ to 70$)$ & 80 (57 to 103$)$ & 94 (71 to 117$)$ & $<0.05$ \\
\hline
\end{tabular}

*Wilcoxon's rank sum test of differences between changes before and after treatment. volume greater than $200 \mathrm{ml}$ on catheterisation, one had a bladder tumour, one had a urethral stricture, and one had an obstructing middle lobe of the prostate. Two patients failed to attend for follow up so that 40 patients were reviewed at three months: 22 (mean age 63.7 years) had received microwave treatment and 18 (mean age 62.6 years) sham treatment. The two groups were matched for age and overall symptom severity, and there were no significant differences in their initial daytime frequency, peak flow rates, and residual volumes.

The table shows that the patients who received microwave treatment showed greater improvement than the controls in all the symptom scores and their mean total symptom score fell from 30 (indicating severe symptoms requiring medical intervention) to 11 (mild symptoms not requiring treatment), which was is significantly better than the improvement in the $\overrightarrow{0}$ control group's score (from 31 to 26$)(p<0.001)$. Peak urine flow rates and residual urine volumes were $\bar{\omega}$ improved by microwave treatment whereas they were worse after sham treatment.

A successful outcome was defined as a reduction of $\omega$ the total symptom score by $50 \%$ or more. On this basis 18 of the 22 patients who received microwave treat- $\infty$ ment were successfully treated while two experienced $\infty$ a $20-50 \%$ reduction in symptom scores and two $\vec{\sim}$ experienced little or no benefit ( $<20 \%$ reduction). Of $\stackrel{\omega}{\omega}$ the 18 patients in the control group, three experienced 0 a reduction of $>50 \%$, three a reduction of $20-50 \%$, and 12 a reduction of $<20 \%$. Perhaps the most disabling $\mathrm{F}$ symptoms of prostatism are frequency, urgency, and nocturia. Among the patients given microwave treatment the mean daytime frequency fell from 9.4 voids a $\overrightarrow{0}$

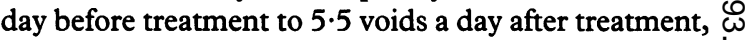
the number of patients experiencing urgency at least $\square$ half the time fell from 15 to three, and the number having to void more than once a night fell from 20 to 10. In the control group there was no improvement in daytime frequency or nocturia and the number $\stackrel{\odot}{\perp}$

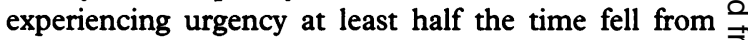
nine to six. The mean peak flow rate increased by $\geqslant 2.0 \mathrm{ml} / \mathrm{s}$ in 15 patients who had received microwave treatment but in only three patients who had received sham treatment.

The treatment was generally well tolerated: all patients were discharged home the same day, and none suffered urinary retention. Bladder spasm during treatment occurred in 14 patients receiving microwave treatment and 11 patients receiving sham treatment. $\delta$ This discomfort was most severe during the early part of treatment, but only one patient required sedation $ᄋ$ in addition to the topical lignocaine gel. During $N$ microwave treatment 18 patients reported only mild $\supset$ or moderate discomfort, but two experienced some tenesmus during the early part of their treatment. $\mathrm{N}$ After microwave treatment seven patients had some $N$ bloodstained urethral discharge, dysuria, urgency, or $\omega$ frequency. These problems were mild and self limiting and all resolved within 48 hours. No patient was $\varrho$ rendered impotent by microwave treatment, all patients remained capable of antegrade ejaculation, $\stackrel{+}{+}$ and three patients reported an improvement in their sex lives. Symptoms began to improve 7-10 days after $\underset{\mathbb{D}}{\vec{D}}$ treatment and continued to improve for up to three $\frac{?}{\mathbb{D}}$ months before reaching a plateau (we have now $\stackrel{2}{\circ}$ followed up these patients for six months).

Patients were also asked for their perception of the 8 effects of their treatment. Of the 22 patients who had received microwave treatment, 17 said that they felt $0 \overline{0}$

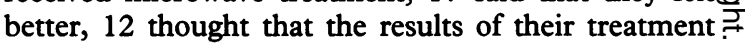
had been better than they had expected, and 20 said they would recommend the treatment to others with similar prostatic symptoms. Of the 18 patients who had received sham treatment, nine said that they felt 
better, three thought that the outcome of their treatment had been better than they had expected, and 15 said they would recommend this treatment to others with a similar problem. The patients were also asked which treatment they thought they had received: 19 of those who had received microwave treatment answered correctly, while half the patients who had received sham treatment thought they had received a real treatment.

\section{Discussion}

We have shown that a single transurethral microwave treatment produced a significantly greater improvement in symptom scores in patients with benign prostatic hypertrophy than sham treatment and improved peak urine flow rates and residual urine volumes. There seemed to be some placebo effect because nine of the patients in the control group thought that their symptoms had improved whereas only six had experienced a reduction of $20 \%$ or more in their symptom scores (and only three had experienced a reduction of $50 \%$ or more). Assessment by semiobjective symptom scores therefore seems to minimise the placebo effect.

In early uncontrolled trials of microwave treatmen the transrectal route was used with an anteriorly directed and cooled transmitter delivering calculated temperatures of $43-45^{\circ} \mathrm{C}$ within the prostate..$^{1617}$ Multiple treatments were required (up to 18) and significant symptomatic improvement occurred in $25-41 \%$ of patients, although formal symptom scores were not recorded. These poor success rates (our success rate was $82 \%$ ) are probably explained by two factors: firstly, the transrectal route is less effective than the transurethral route ${ }^{20}$; and, secondly, the patients in these trials were unselected, and we now know that patients with large prostates or obstructing middle lobes respond poorly to microwave treatment.

Transrectal treatment has been largely superseded by transurethral devices so that most of the heat is delivered to the periurethral prostatic tissue with the rectal mucosa receiving relatively low heat. In a tria comparing the effectiveness of multiple transrectal and transurethral microwave treatments symptom scores were significantly improved in $79 \%$ of patients who received transurethral treatments compared with $41 \%$ of patients who received transrectal treatments. ${ }^{20}$ In uncontrolled studies of transurethral microwave treatments, with different machines and multiple treatments, appreciable symptomatic improvement (greater than $50 \%$ reduction in symptom scores) was reported in $70-89 \%$ of patients, daytime frequency was improved by $21-28 \%$, nocturia was improved by $43-81 \%$, residual volumes fell by $49-81 \%$, and flow rates increased by $4 \cdot 9-6 \cdot 2 \mathrm{ml} / \mathrm{s}^{18-20}$

Laduc et al performed a trial of single session microwave treatment using a transurethral device with a water cooled antenna and reported a substantial improvement in $63 \%$ of patients, a $21 \%$ reduction in residual urine volumes, and an increase in mean peak flow rates of $1.0 \mathrm{ml} / \mathrm{s}^{21} \mathrm{~A}$ recent study compared a single transurethral microwave treatment, again using a water cooled antenna, with sham treatment. ${ }^{22}$ The microwave treatment resulted in a $70 \%$ improvement in symptom scores, an increase in peak flow rates of $4.5 \mathrm{ml} / \mathrm{s}$, and a $92 \%$ reduction in residual volumes; there was no significant improvement after sham treatment. Our results with a single transurethral treatment were similar: we found a $63 \%$ improvement in symptom scores, an increase in peak flow rates of 2.3 $\mathrm{ml} / \mathrm{s}$, and a $50 \%$ reduction in residual volumes. We also found no significant improvement after sham treatment.

Transurethral microwave treatment compares favourably with prostatectomy in terms of symptomatic improvement. Neal et al reported that $75 \%$ of men obtained good symptomatic relief after elective prostatectomy but $12 \%$ still complained of substantial hesitancy, $39 \%$ of dribbling after micturition, and $32 \%$ of urgency. ${ }^{6}$ In our study $40 \%$ of patients still had some hesitancy after microwave treatment, but only $7 \%$ had substantial dribbling after micturition and $10 \%$ had substantial urgency (a symptom was defined as substantial if it was present $50 \%$ or more of the time).

Microwave treatment is free of complications compared with prostatectomy. After surgery incontinence occurs in up to $4 \%$ of patients, and $5 \%$ are rendered impotent, ${ }^{7}$ but neither of these problems occurred after microwave treatment. Over $60 \%$ of men suffer retrograde ejaculation after prostatectomy ${ }^{8}$ whereas microwave treatment preserves antegrade ejaculation. This is particularly important in younger, sexually active men. Other side effects, although seldom severe, do occur after surgery and include clot retention, urethral stricture, and postoperative haemorrhage requiring blood transfusion; these problems do not occur after microwave treatment. Transurethral prostatectomy, however, remains the standard against which all other treatments for benign prostatic hypertrophy should be judged, and no microwave treatment has been shown to relieve obstruction to the same degree: increases in flow rates after microwave treatment are modest compared with the increases seen after surgery. Prostatectomy remains the treatment of choice for patients with a very large gland and a decompensated bladder with residual urine volumes in excess of $200 \mathrm{ml}$. Preliminary studies (CJB and RO'NW, unpublished data) showed microwave treatment to be relatively ineffective for such patients.

As microwave treatment is relatively new the length of time patients will experience relief from symptoms is unknown, but we have followed up patients in an earlier trial for two years and there has been no deterioration in symptoms. Longer term follow up is required to compare the results with the $20 \%$ reoperation rate within eight years after prostatectomy.9

The mode of action of the treatment is obscure. The increase in peak flow rates after treatment was similar to that seen after $\alpha$ blockade, ${ }^{11}$ although microwave treatment resulted in a greater improvement in symptoms. The heat generated during microwave treatment possibly destroys $\alpha$ receptors and sensory nerve endings at the bladder neck and in the proximal prostatic urethra. This remains hypothetical, however, and further studies are required.

We showed that a single transurethral microwave treatment given to outpatients was safe, effective, and well tolerated by most patients. It caused minimal disruption to the patients and preserved sexual function and antegrade ejaculation. These factors and the logistical problems of treating the increasing numbers of patients with prostatism mean that transurethral microwave treatment is a highly promising option for the treatment of prostatism.

1 Lytton B, Emery JM, Harvard BM. The incidence of benign prostatic obstruction. F Urol 1968;99:639-45.

2 Glynn RJ, Campion EW, Bouchard GR, Silbert JE. The development of benign prostatic hyperplasia among volunteers in the normative ageing study. Am $\mathcal{F}$ Epidemiol 1985;121:78-90.

3 Berry SJ. The development of human prostatic hyperplasia with age. $f$ Urol 1984;132:474-9.

4 Chilton CP, Morgan RJ, England HR, Paris AMI, Blandy JP. A clinical evaluation of the results of transurethral resection of the prostate. $B r \mathcal{F}$ Urol 1978;50:542-6.

5 Bruskewitz RC, Larsen EH, Madsen PO, Dorflinger T. 3 year follow up of urinary symptoms after transurethral resection of the prostate. $f$ Urol 1986;136:613-5.

6 Neal DE, Ramsden PD, Sharples L, Smith A, Powell PH, Styles RA, et at. Outcome of elective prostatectomy. $B M \mathcal{F}^{1989 ; 299: 762-7}$

7 Fowler FJ, Wennenberg JE, Timothy RP, Barry MJ, Mulley AJ, Hanley D. Symptom status and quality of life following prostatectomy. $\mathscr{F} A M A$ 1988;259:3018-22.

8 Neal DE. Bladder outflow obstruction and the outcome of surgery. In: Hendry 
WF, ed. Recent advances in urology/andrology. Vol 5. Edinburgh: Churchill Livingstone, 1991:149-65.

9 Wennenberg JE, Roos N, Sola L, Schori A, Jaffe R. Use of claims data systems to evaluate health care outcomes. $\Im A M A$ 1987;257:933-6.

10 Bosch RLJH, Griffiths DJ, Blom JHM, Schroeder FH. Treatment of benign prostatic hyperplasia by androgen deprivation; effects on prostate size and urodynamic parameters. $\mathcal{F}$ Urol 1989;141:68-72.

11 Caine M, Perlberg S, Meretyk SA. A placebo controlled double blind study of the effects of phenoxybenzamine in benign prostatic obstruction. $\mathrm{Br} f \mathrm{Urol}$ 1978;50:551-4.

12 Reddy PK, Wasserman N, Casteneda F, Castenedazuniga WR. Balloon dilatation of the prostate for the treatment of benign hyperplasia. dilatation of the prostate for the
Urol Clin North Am 1988;15:529-33.

13 Klein LA, Lemming B. Balloon dilatation for prostatic obstruction: long term follow up. Urology 1989;33:198-201.

14 Vincente J, Salvador J, Chechile G. Spiral urethral prosthesis as an alternative to surgery in high risk patients with benign prostatic hyperplasia: a prospective study. I Urol 1989;142:1504-6.

15 Kirby RS, Bryan J, Eardley I, Christmas TJ, Liu S, Holmes SAV, et al. Finasteride in the treatment of benign prostatic hyperplasia: a urodynamic evaluation. Br J Unol 1992;70:65-72.

16 Yerushalmi A, Fishelovitz Y, Singer D, Reiner I, Arielly J, Abramovici Y, et al. Localised deep microwave hyperthermia in the treatment of poo operative risk patients with benign prostatic hyperplasia. $f$ Urol 1985;133: 873-6.

17 Servadio C, Leib Z, Lev A. Diseases of the prostate treated by local microwave hyperthemia. Urology 1987;30:97-9.

18 Sapozink MD, Boyd SD, Astrahan MA, Jozsef G, Petrovitch S. Transurethral hyperthermia for benign prostatic hyperplasia: preliminary clinical results. fUrol 1990;143:944-9.

19 Baert L, Ameye F, Vandenhove J, Lauweryns J, Astrahan M, Petrovich Z. Transurethral microwave hyperthermia for benign prostatic hyperplasia: preliminary clinical and pathological results. $f$ Urol 1990;144:1383-7.

20 Stawarz B, Szmigielski J, Ogrodnik J, Astrahan M, Petrovich Z. A compariso of transurethral and transrectal hyperthermia in poor surgical risk benign of transurethral and transrectal hyperthermia in poor

21 Laduc R, Bloem FAG, Debruyn FMJ. Transurethral microwave thermotherapy (TUMT) in the treatment of patients with benign prostatic hyperplasia. European Urology Update 1992;1:42-7.

22 Ogden CW, Reddy P, Johnson H, Ramsay JWA, Carter SStC. Sham versus transurethral microwave thermotherapy in patients with symptoms of benign prostatic bladder outflow obstruction. Lancet 1993;341:14-7.

(Accepted 2 March 1993)

\title{
Vertically transmitted HIV infection in the British Isles
}

\author{
A E Ades, Clare F Davison, Fiona J Holland, Diana M Gibb, Christopher N Hudson, Angus Nicholl, \\ David Goldberg, C S Peckham
}

Department of

Epidemiology and

Biostatistics, Division of

Public Health, Institute of

Child Health, London

WC1N 1EH

A E Ades, senior lecturer in biostatistics

Clare F Davison, lecturer in

epidemiology

Fiona J Holland, research

programmer

Diana M Gibb, senior lecturer in epidemiology

C S Peckham, professor of

paediatric epidemiology

Department of Obstetrics and Gynaecology, St

Bartholomew's Hospital,

London EC1A 7BE

Christopher N Hudson,

professor of obstetrics

Public Health Laboratory

Service AIDS Centre,

Communicable Diseases

Surveillance Centre,

London NW9 5EQ

Angus Nicholl, consultant

epidemiologist

Communicable Disease

(Scotland) Unit, Ruchill

Hospital, Glasgow

G20 9NB

David Goldberg, honorary

senior lecturer

Correspondence to:

Dr Ades.

BMF 1993;306:1296-9

\section{Abstract}

Objective-To describe the epidemiology of vertically acquired HIV infection in the British Isles, the level of underreporting, the vertical transmission rate, and clinical spectrum of paediatric AIDS.

Design-Confidential, linked registers based on reporting from obstetricians and paediatricians; anonymous unlinked neonatal HIV serosurveys.

Setting-British Isles.

infected or not. The paediatric and obstetric schemes are linked making prospective data analyses possible

Routine paediatric HIV surveillance tables are published quarterly in the Communicable Disease Report'; AIDS Scotland'; (ANSWER) Communicable Diseases Scotland Weekly Reports; and the National Study of HIV in Pregnancy quarterly newsletters. ${ }^{7}$ We report here on the descriptive epidemiology and ascertainment of vertically acquired paediatric HIV infection in the British Isles, the vertical transmission rate, and the clinical spectrum of reported AIDS. infection.

Main outcome measures-Trends in HIV infection and vertical transmission rate.

Results-In Scotland and the Irish Republic, where most maternal HIV infection is related to drug misuse, the annual number of reports of children born to infected mothers has fallen since 1989. In England and Wales nearly half of maternal infections have been acquired overseas, and the number of children born to these women, and to women who became infected in Britain, is increasing. In south east England the proportion of live births to women whose infection was identified before delivery was only $17 \%(50 / 287)$, compared with $68 \%(26 / 38)$ in Scotland. The vertical transmission rate was $13.7 \%$ $(23 / 168)$, and $23 \%$ of infected children developed AIDS in the first year of life. $41 \%$ (38/92) of children born to infected mothers who were ascertained after delivery were breast fed, compared with $5 \%$ (12/236) of those ascertained before delivery.

Conclusions-The incidence of vertically transmitted HIV infection is increasing in England and Wales. More extensive antenatal testing would enable infected women to be counselled against breast feeding, which could prevent a substantial proportion of vertical transmission in some areas, and would increase opportunities for early diagnosis and treatment of infected children.

\section{Introduction}

Active surveillance of AIDS in children in the British Isles began in 1986 through the British Paediatric Surveillance Unit. ${ }^{1}$ In 1989 reporting of pregnant seropositive women was initiated in collaboration with the Royal College of Obstetricians and Gynaecologists, ${ }^{23}$ and paediatric surveillance was extended to include all HIV positive children, whether

\section{Methods}

Obstetricians report pregnancies in HIV positive women through a scheme run in collaboration with the Royal College of Obstetricians and Gynaecologists. ${ }^{3}$ A designated person from each obstetric unit in the British Isles is asked to return a quarterly report card on behalf of the unit, including nil returns if there are no cases to notify. Reports are followed up with a standard questionnaire to obtain information on outcome of pregnancy.

Paediatricians are asked to notify The British Paediatric Surveillance Unit of children who are infected and those of indeterminate infection status each month. ${ }^{8}$ The reporting identifies children with AIDS; those with HIV infection as indicated by virus culture, polymerase chain reaction, antigen, or persistence of antibody beyond 18 months; and children of indeterminate status, defined as antibody positive but under 18 months. Cases are also reported directly to the coordinating centre. Reports are followed up annually with a standard questionnaire to determine infection status and clinical progress. Paediatricians are requested to report development of AIDS and death in the interim. We used the Centre for Disease Control definition of AIDS with minor modifications (see footnote of table III). ${ }^{\circ}$

The obstetric and paediatric schemes have over $90 \%$ compliance. ${ }^{38}$ Cases first identified before 1989 were reported retrospectively. Laboratory reports of HIV antibody positive children forwarded to the Communicable Disease Surveillance Centre and the Communicable Diseases (Scotland) Unit constitute a third scource of data. Data from all sources are combined into a single dataset. All clinical reports are 